\title{
Inflammatory Polyarthritis in a Patient with Psoriasis: Is It Psoriatic Arthritis or Rheumatoid Arthrirtis?
}

\author{
Kwang-Hoon Lee, Myoung-Kyun Son, You-Jung Ha, Sang-Tae Choi, Sang-Won Lee, \\ Yong-Beom Park, and Soo-Kon Lee
}

Division of Rheumatology, Department of Internal Medicine, Yonsei University College of Medicine, Seoul, Korea

Psoriatic arthritis (PsA) is an inflammatory arthritis associated with psoriasis. There are no generally accepted diagnostic criteria for PsA. Indeed, the diagnosis of this inflammatory arthritis is made by exclusion of other possible diseases and based upon immunologic, radiologic, and clinical features which are consistent with the diagnosis. Inflammatory arthritis in a patient with psoriasis can be an important clue for the diagnosis of PsA, but the possibility for diagnosis of other inflammatory arthritides ever remains. Herein we report a case of a female patient who was not diagnosed with PsA, but with rheumatoid arthritis, even though she had psoriasis. (Korean $\mathbf{J}$ Intern Med 2010;25:224-226)

Keywords: Psoriasis; Arthritis, rheumatoid; Arthritis, psoriatic

\section{INTRODUCTION}

Psoriatic arthritis (PsA) is an inflammatory arthritis associated with psoriasis. The prevalence of PsA among patients with psoriasis ranges from $3 \%$ to $30 \%$ [1-3]. Currently, the diagnosis of PsA is based on clinical, radiologic, and immunologic features which are consistent with the diagnosis of psoriatic arthritis, rather than other inflammatory arthritides, because there are no generally accepted criteria for the classification of PsA. Sometimes, it is difficult to differentiate PsA from rheumatoid arthritis (RA) when the disease is manifested by a polyarticular pattern and the rheumatoid factor (RF) is positive.

\section{CASE REPORT}

A 41-year-old woman was admitted to our hospital because of multiple joint pains and swelling of 1 month duration. She had been diagnosed with psoriasis since 10 years of age. Two months previously, her skin lesions were exacerbated and she had multiple erythematous patches and pinhead-sized pustules distributed over her entire body. She was treated with acitretin and narrow-band ultraviolet $\mathrm{B}$ radiation, but her skin lesions did not improve. After the use of oral prednisolone (30 mg/day), there was a slight improvement in her skin lesions. The prednisolone was tapered over 2 weeks. Several days after cessation of the prednisolone, she began to complain of pain and swelling involving multiple joints, which led to her visit to our office for evaluation. On physical examination, there were multiple tender and swollen joints including the right shoulder, the elbows, the wrists, the proximal interphalangeal joints, the knees, and the ankles, but there was no involvement of the distal interphalangeal (DIP) joints nor findings of nail dystrophy (Fig. 1). Laboratory findings revealed a white blood cell count of $11,450 / \mu \mathrm{L}$, a hemoglobin level of $11.9 \mathrm{~g} / \mathrm{dL}$, a platelet count of 395,000 $/ \mu \mathrm{L}$, an erythrocyte sedimentation rate of $120 \mathrm{~mm} / \mathrm{hr}$, a C-reactive protein level of $31.7 \mathrm{mg} / \mathrm{dL}$, a RF level of 966 $\mathrm{IU} / \mathrm{mL}$, and an anti-cyclic citrullinated peptide antibody (anti-CCP Ab) titer of $229.3 \mathrm{U} / \mathrm{mL}$. A whole body bone 

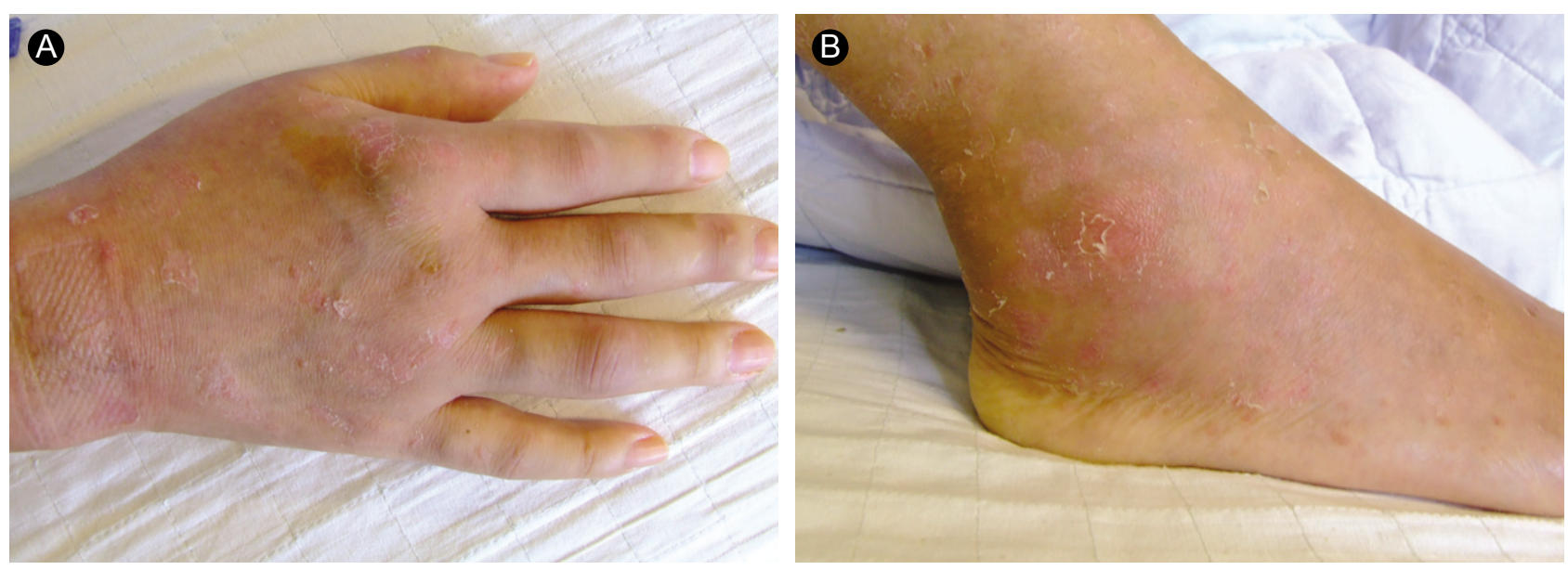

Figure 1. Photographs of the hand and foot. There are multiple erythematous patches suggesting psoriasis on the hand and foot. (A) Shows swellings on Proximal interphalangeal, Metacarpophalangeal, and wrist joints. (B) Shows swelling of the ankle joints.
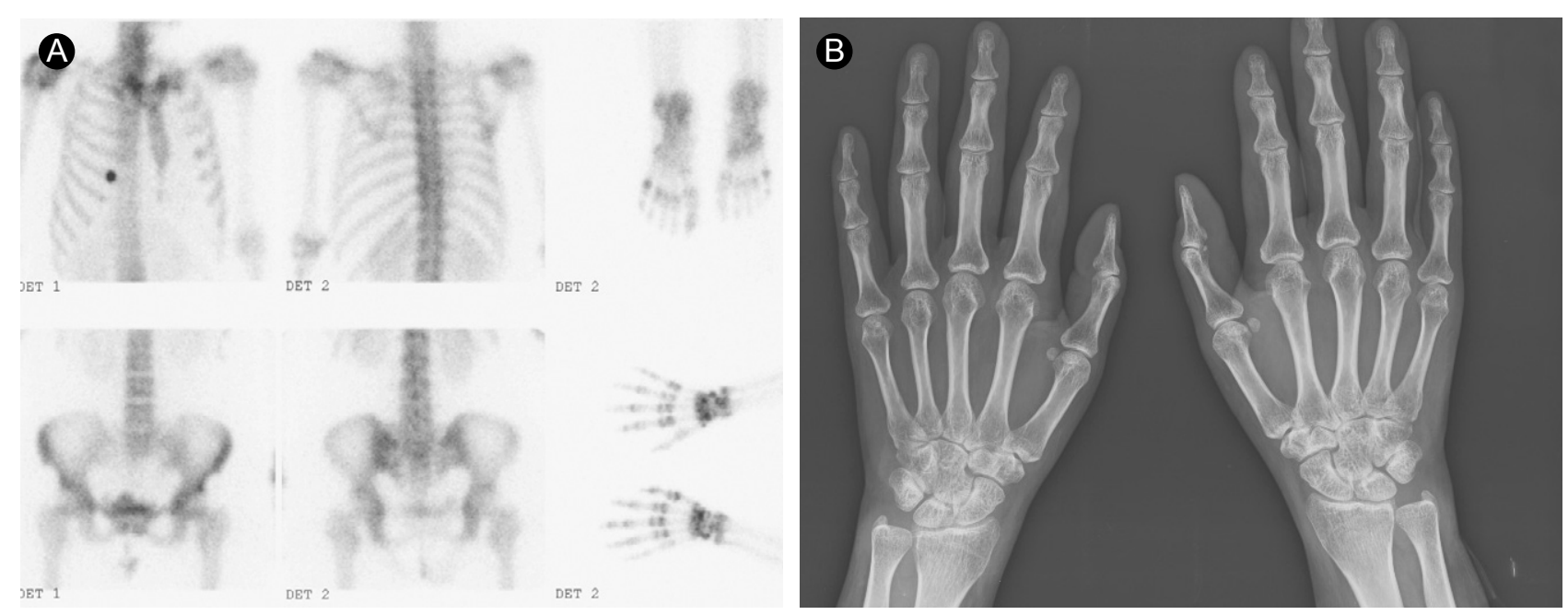

Figure 2. Radiographic assessment. (A) Whole body bone scan shows multifocal increased uptakes on bilateral wrist and finger joints, suggesting arthritic changes. (B) The plain radiograph of both hands showed no grossly abnormal findings.

scan showed multiple increased uptakes in bilateral $5^{\text {th }}$ metatarsal phalangeal joints, wrists, and finger joints, suggesting the presence of inflammatory arthritis, but plain X-ray studies showed no significant bony abnormalities, including periostitis and marginal bone erosion (Fig. 2). On the basis of clinical features such as symmetric inflammatory polyarthritis of the hand joints involving more than 3 joints and the presence of RF and anti-CCP Ab, we assigned the diagnosis of RA with psoriasis rather than PsA. She had began treatment with methotrexate $(7.5 \mathrm{mg} / \mathrm{wk})$, sulfasalazine ( $2 \mathrm{~g} /$ day), hydroxychloroquine (200 $\mathrm{mg} /$ day), prednisolone (10 $\mathrm{mg} /$ day), and meloxicam ( $15 \mathrm{mg} /$ day). She is now followed in the regular outpatient department and her pain and swelling of the joints are improving.

\section{DISCUSSION}

There are currently no diagnostic criteria for PsA; the diagnosis of PsA is made clinically. Moll and Wright [4] have described five clinical patterns of joint involvement in PsA: 1) distal arthritis (involvement of the DIP joints), 2) asymmetric oligoarthritis, 3) symmetric polyarthritis, 4) arthritis mutilans, and 5) spondyloarthropathy. While distal arthritis and arthritis mutilans are specific for PsA, polyarthritis is thought to be more frequent in PsA [5]. Sometimes, PsA presenting with a polyarticular pattern is clinically indistinguishable from RA. Generally, PsA can be discriminated from RA by physical findings, such as the presence of DIP joint involvement, an asymmetric pattern of arthritis, spondyloarthropathy, dactylitis, and nail 
dystrophy. However, the absence of these clinical features does not exclude PsA as a possible diagnosis. RF exists in $2 \%$ to $10 \%$ of patients with PsA. Low titers of anti-nuclear antibodies are also found in nearly one-half of patients with PsA; clinically significant titers (i.e., $\geq 1: 80$ ) exist in $14 \%$ of patients with PsA [6]. Anti-CCP Ab, with a high specificity for RA, are widely used in distinguishing RA from other disease conditions [7]. However, anti-CCP Ab are found in $8 \%$ to $16 \%$ of patients with PsA, especially in those with erosive and polyarticular disease, and anti-CCP $\mathrm{Ab}$ are also found in those patients with psoriasis alone [8]. Therefore, the presence of anti-CCP Ab do not exclude PsA from a possible alternative diagnosis. In this patient, we made the diagnosis of RA because she had morning stiffness, arthritis involving more than 3 joints, arthritis of the hand, RF positivity, and seropositivity for antiCCP Ab. However, we could not verfiy that she did not have PsA because there is no generally agreed upon diagnostic criteria for PsA.

Several criteria for PsA have been suggested. Recently, new diagnostic criteria have been suggested by Taylor et al. [9], the so-called CASPAR (classification criteria for psoriatic arthritis) criteria, the sensitivity and specificity of which are $91.4 \%$ and $98.7 \%$ respectively. The criteria consist of established inflammatory articular disease with at least a sum of three points from the following: current psoriasis (2 points), a history of psoriasis (1 point), a family history of psoriasis (unless current psoriasis was present or there was a history of psoriasis; 1 point), dactylitis (1 point), juxtaarticular new bone formation (1 point), RF negativity (1 point), and nail dystrophy (1 point). In the case of our patient, she had inflammatory articular disease and psoriasis. Therefore, according to the CASPAR criteria, she could not be diagnosed with PsA because she scored 2 points only.

It is easy to think that a patient with both psoriasis and inflammatory arthritis may have PsA. However it should be noted that other forms of arthritis can be present in the setting of psoriasis and inflammatory arthritis. Assessing serologic markers, such as RF and anti-CCP Ab, can be of help in the differential diagnosis, but it should be noted that the laboratory studies also have limitations, i.e., false positivity. It is sometimes difficult to diagnose a patient with PsA due to a lack of internationally accepted criteria. Newly developed criteria, such as the CASPAR criteria, can facilitate the diagnosis of PsA. We hope that appropriate diagnostic criteria will be accepted for the diagnosis of PsA in the future.

\section{Conflict of interest}

No potential conflict of interest relevant to this article was reported.

\section{REFERENCES}

1. Shbeeb M, Uramoto KM, Gibson LE, O'Fallon WM, Gabriel SE. The epidemiology of psoriatic arthritis in Olmsted County, Minnesota, USA, 1982-1991. J Rheumatol 2000;27:1247-1250.

2. Zachariae $H$. Prevalence of joint disease in patients with psoriasis: implications for therapy. Am J Clin Dermatol 2003;4:441-447.

3. Gelfand JM, Gladman DD, Mease PJ, et al. Epidemiology of psoriatic arthritis in the population of the United States. J Am Acad Dermatol 2005;53:573.

4. Moll JM, Wright V. Psoriatic arthritis. Semin Arthritis Rheum 1973;3:55-78.

5. Gladman DD. Current concepts in psoriatic arthritis. Curr Opin Rheumatol 2002;14:361-366.

6. Bizzaro N. Antibodies to citrullinated peptides: a significant step forward in the early diagnosis of rheumatoid arthritis. Clin Chem Lab Med 2007;45:150-157.

7. Johnson SR, Schentag CT, Gladman DD. Autoantibodies in biological agent naïve patients with psoriatic arthritis. Ann Rheum Dis 2005;64:770-772.

8. Alenius GM, Berglin E, Rantapaa Dahlqvist S. Antibodies against cyclic citrullinated peptide (CCP) in psoriatic patients with or without joint inflammation. Ann Rheum Dis 2006;65:398-400.

9. Taylor W, Gladman D, Helliwell P, et al. Classification criteria for psoriatic arthritis: development of new criteria from a large international study. Arthritis Rheum 2006;54:2665-2673. 